\title{
Use of Levomepromazine for the Treatment of
} Emergence Agitation in Patients Receiving Mechanical Ventilation for SARS-CoV-2 Related Acute Respiratory Distress Syndrome

\author{
Declercq PL ${ }^{1 *}$, Cousin $T^{2}$, Eraldi J P ${ }^{1}$, Marchalot A ${ }^{1}$, \\ Bougerol $F^{1}$, Beuzelin $M^{1}$, Gélinotte $S^{1}$ and Rigaud \\ JP 1 \\ 'Service de Médecine Intensive Réanimation, Centre \\ Hospitalier de Dieppe, France \\ ${ }^{2}$ Service de Pharmacie, Centre Hospitalier de Dieppe, \\ France \\ *Corresponding author: Pierre-Louis Declercq, \\ Service de Médecine Intensive Réanimation, Centre \\ Hospitalier de Dieppe, Avenue Pasteur, 76202 Dieppe, \\ France
}

Received: September 24, 2021; Accepted: October 26, 2021; Published: November 02, 2021

\begin{abstract}
Purpose: To report our experience with the use of Levomepromazine (LMZ) for the treatment of delirium in patients treated for Acute Respiratory Distress Syndrome (ARDS) due to COVID-19.

Methods: Retrospective series of patients admitted to the Intensive Care Unit (ICU) of a non-academic hospital and who requiring invasive mechanical ventilation for the treatment of ARDS due to SARS-CoV-2. Infection was confirmed by Reverse Transcriptase Polymerase Chain Reaction (RT-PCR) testing, and patients presented delirium after withdrawal of neuromuscular blockade or sedation lightening treated with LMZ on top of other treatment.
\end{abstract}

Results: Out of 34 ARDS patients admitted from 22 March 2020 to 23 January 2021, $11(32.3 \%)$ died before neuromuscular blockade withdrawal, and of the remaining 23,16 were treated with LMZ. The median time to initiation of LMZ was 6 days (interquartile, 5 to 8 ) from the start of sedation, and median duration of LMZ treatment was 5 (1 to 28) days. Median sedation score (as assessed by the Sedation Agitation Scale (SAS)) was 2 (1-4) at initiation and 3 (2-4) at discontinuation of LMZ, while median analgesia scores (Behavioral Pain Scale (BPS)) were respectively $3(3-5)$ and $4(3-5)$. Of the 16 patients treated with $L M Z, 12$ were weaned from mechanical ventilation immediately following discontinuation, without recurrence of delirium. No adverse effects related to LMZ use were observed. The 4 patients who could not be weaned from mechanical ventilation following LMZ required new neuromuscular blockade.

Conclusions: Based on this retrospective series, LMZ seems to be useful for the management of delirium occurring after withdrawal of neuromuscular blockade or lightening of sedation in patients with ARDS due to SARS-CoV-2.

Keywords: Levomepromazine; COVID-19; SARS-CoV-2; Delirium; Acute respiratory distress syndrome; mechanical ventilation

\section{Introduction}

The ongoing pandemic caused by the Severe Acute Respiratory Coronavirus 2 (SARS-CoV-2), and the resulting pneumonia termed COVID-19, has led to a massive influx of patients to intensive care units worldwide. The severity of the disease is largely due to the respiratory tropism of the virus, and the tendency of infected individuals to progress to Acute Respiratory Distress Syndrome (ARDS). In addition to the respiratory symptoms, the high frequency of neurological features related to COVID-19 is noteworthy such as delirium. Delirium seems to be frequent in patients invasively ventilated for the treatment of ARDS caused by SARS-CoV-2 and identified after the discontinuation of neuromuscular blockade [1]. In a study by Helms et al, delirium was diagnosed in 97 (79.5\%) of 122 patients with ARDS mainly in its hyperactive form. The authors underlined the high frequency of complications associated with these neurological features, notably more auto-extubations, a longer duration of mechanical ventilation, a longer hospital stay, and higher mortality [2].

The origin of this agitated delirium is likely multifactorial, including damage to cells of the Central Nervous System (CNS) linked to the viral infection, inflammation of the CNS, organ failure, or the treatments used to achieve sedation-analgesia [3]. It is clearly necessary to treatment delirium rapidly, with a view to reducing the associated risk of early and late complications [4], and also to enable weaning from ventilation. No specific treatment is currently recommended for the management of delirium, largely due to a lack of efficacy data [4]. Haloperidol, a typical antipsychotic, is widely used in this indication, but without formal proof of its efficacy [5]. Atypical antipsychotics are also used, while more recently, dexmedetomidine, a sedative alpha-2 agonist, was evaluated and showed promising results [6].

We report here our experience with the use of Levomepromazine (LMZ) for the treatment of delirium in patients treated for ARDS due to COVID-19. 


\section{Methods}

We conducted a retrospective study in 1 ICU from a nonacademic hospital, from 22 March 2020 to 23 January 2021. All patients who required invasive mechanical ventilation for the treatment of ARDS due to SARS-CoV-2 were selected if the infection was confirmed by Reverse Transcriptase Polymerase Chain Reaction (RT-PCR) testing. Only the patients who presented agitation after neuromuscular blockade withdrawal or sedation lightening were eligible if they received intravenous LMZ.

This study did not require written informed consent in accordance with French legislation and as confirmed by the Ethics Committee CPP Est I (Dijon University Hospital, France).

\section{Results}

During the study period, 34 patients were admitted for the treatment of ARDS due to SARS-CoV-2. Eleven patients (32.3\%) died before neuromuscular blockade was discontinued or before sedation was lightened. Among the remaining 23 patients, 16 were treated with LMZ, which was administered systematically by the intravenous route, in continuous flow using an electric syringe pump, and preceded by an intravenous bolus ( 25 or $50 \mathrm{mg}$ ) over 30 to 60 minutes if a swift sedative effect was needed. The indications for LMZ use were agitation and/or prevention of the patient fighting the ventilator, after a failed attempt at withdrawal of neuromuscular blockade or lightening of sedation, due to a pathological neurological state. LMZ was used on top of other treatments (midazolam, sufentanil, propofol) to achieve sedation and avoid complications related to agitation. The daily dose of the other molecules was rapidly reduced until complete discontinuation if the patient's respiratory status was amenable to their withdrawal.

Characteristics of patients concerning the LMZ use and outcomes are resumed in Table 1. The median time to initiation of LMZ was 6 days (interquartile, 5 to 8 ) from the start of sedation and invasive mechanical ventilation. The median duration of LMZ treatment was 5 days (1-28). Of the 16 patients treated with LMZ, 12 were weaned from mechanical ventilation immediately following discontinuation, without recurrence of delirium. In these patients, the median duration of LMZ treatment was 3.5 days (1-8). No adverse effects related to LMZ use were observed. The four patients who could not be weaned from mechanical ventilation following LMZ initiation required new neuromuscular blockade due to deterioration of their respiratory status.

\section{Discussion}

Based on this retrospective series, LMZ seems to be useful for the management of delirium occurring after withdrawal of neuromuscular blockade or lightening of sedation in patients with ARDS due to SARS-CoV-2. In our study, the use of LMZ enabled progressive withdrawal of conventional sedatives (benzodiazepines, opioids), enabling weaning from mechanical ventilation. However, the absence of a correlation with the SAS and BPS scores should be noted, as these scores were not in favour of a state of agitation or discomfort that would require reinforced sedation in agitated patients. The scores recorded in this study were the latest scores available prior to introduction of LMZ, while the patients were still
Table 1: Characteristics of patients with SARS-CoV-2 ARDS and delirium.

\begin{tabular}{|l|c|}
\hline \multicolumn{1}{|c|}{ Variables } & All Patients (N=16) \\
\hline Age, years, median (IQR) & $70(56-76)$ \\
\hline Male sex, n (\%) & $23(17-41)$ \\
\hline Median length of ICU stay, days (IQR) & $15(11-24)$ \\
\hline Median duration of mechanical ventilation, days (IQR) & \\
\hline Sedation for ARDS before initiation of LMZ & $6(5-8)$ \\
\hline Median number of days of sedation (IQR) & $16(100)$ \\
\hline Midazolam, n (\%) & $4.5(3-6.75)$ \\
\hline Days of treatment, median (IQR) & $16(100)$ \\
\hline Sufentanil, n (\%) & $5(3-6.75)$ \\
\hline Days of treatment, median (IQR) & $9(56)$ \\
\hline Propofol, n (\%) & $1(0-2)$ \\
\hline Days of treatment, median (IQR) & $2(1-4) / 3(2-4)$ \\
\hline Median SAS score at start/end of LMZ (IQR) & $3(3-5) / 4(3-5)$ \\
\hline Median BPS score at start/end of LMZ (IQR) & $10(62.5)$ \\
\hline Bolus of LMZ, n (\%) & $150(100-200)$ \\
\hline Dose of LMZ infusion, mg/24h, median (IQR) & $12(75)$ \\
\hline nuccessfully weaned from mechanical ventilation, & $(13)$ \\
\hline Death, n(\%) & \\
\hline
\end{tabular}

IQR: Interquartile; ICU: Intensive Care Unit; ARDS: Acute Respiratory Distress Syndrome; LMZ: Levomepromazine; SAS: Sedation Agitation Scale; BPS: Behavioural Pain Scale.

receiving neuromuscular blockade and sedation. The CAM-ICU scale was not used in our study.

LMZ is a phenothiazine neuroleptic agent that has been used for several decades for its antipsychotic effects, ascribed to its antidopaminergic properties. However, the antipsychotic effects are less pronounced than its adrenolytic, anticholinergic and antihistamine activity [7]. It is a potent sedative, which has led to its use in states of agitation due to psychiatric disorders, and it also seems to have an analgesic effect that differs from that of opioids, with the result that LMZ is also used for analgesia [7].

The use of LMZ for the treatment of agitation in the ICU has never been reported in adults, but two series of pediatric patients described the use of LMZ by the oral route for refractory agitation or difficult sedation, with satisfactory results $[8,9]$. To date, there have been no reports of intravenous administration of LMZ. The routes of administration recommended by the manufacturer (Sanofi Aventis) and validated by the French agency for the safety of medicinal products (Agence Nationale de Sécurité du Médicament, ANSM), are the intramuscular and oral routes. Nevertheless no adverse effects were observed in our series with intravenous administration. Haloperidol, another typical antipsychotic, was tested in a randomized, doubleblind, placebo-controlled trial in 192 patients in the ICU with intravenous administration, without any noteworthy adverse effects [5]. Intravenous administration of LMZ makes it possible to achieve a therapeutic plasma concentration more quickly than by the oral route, by avoiding the first pass hepatic metabolism [10].

The efficacy of LMZ is likely linked to its potent sedative effect, mediated by a mechanism of action that is different from that of 
benzodiazepines or opioids. These latter two drug classes may actually contribute to the onset of delirium when used over a prolonged period, as may be the case in patients with ARDS due to SARS-CoV-2.

\section{Conclusion}

Our data suggest that the use of LMZ could be of potential interest for the treatment of agitated delirium occurring during lightening of sedation and withdrawal of neuromuscular blockade, in patients with ARDS due to SARS-CoV-2. Prospective evaluation in clinical trials is warranted.

\section{References}

1. Helms J, Kremer S, Merdji H, Clere-Jehl R, Schenck M, Kummerlen C, et al Neurologic Features in Severe SARS-CoV-2 Infection. N Engl J Med. 2020; 382: $2268-2270$

2. Helms J, Kremer S, Merdji H, Schenck M, Severac F, Clere-Jehl R, et al. Delirium and encephalopathy in severe COVID-19: a cohort analysis of ICU patients. Crit Care. 2020; 24: 491.

3. Kotfis K, Williams Roberson S, Wilson JE, Dabrowski W, Pun BT, Ely EW COVID-19: ICU delirium management during SARS-CoV-2 pandemic. Crit Care. 2020; 24: 176
4. Devlin JW, Skrobik Y, Gelinas C, Needham DM, Slooter AJC, Pandharipande $\mathrm{PP}$, et al. Clinical Practice Guidelines for the Prevention and Management of Pain, Agitation/Sedation, Delirium, Immobility, and Sleep Disruption in Adult Patients in the ICU. Crit Care Med. 2018; 46: e825-e873.

5. Girard TD, Exline MC, Carson SS, Hough CL, Rock P, Gong MN, et al. Haloperidol and Ziprasidone for Treatment of Delirium in Critical Illness. N Engl J Med. 2018; 379: 2506-2516.

6. Reade MC, Eastwood GM, Bellomo R, Bailey M, Bersten A, Cheung B, et al. Effect of Dexmedetomidine Added to Standard Care on Ventilator-Free Time in Patients With Agitated Delirium: A Randomized Clinical Trial. JAMA. 2016; 315: 1460-1468.

7. Patt RB, Proper G, Reddy S. The neuroleptics as adjuvant analgesics. J Pain Symptom Manage. 1994; 9: 446-453.

8. van der Zwaan S, Blankespoor RJ, Wolters AM, Creten C, Leroy PL, Schieveld JN. Additional use of methotrimeprazine for treating refractory agitation in pediatric patients. Intensive Care Med. 2012; 38: 175-176.

9. Snoek A, James P, Arenas-Lopez S, Durward A. Levomepromazine for difficult sedation in pediatric intensive care. J Pediatr Intensive Care. 2014; 3: 53-57.

10. Green B, Pettit T, Faith L, Seaton K. Focus on levomepromazine. Curr Med Res Opin. 2004; 20: 1877-1881. 